\title{
Methamphetamine use and constrictive pericarditis; a rare case of heart in a cage
}

\author{
Mirzaee $\mathrm{S}^{1 *}$, Lau J ${ }^{2}$ and Pincus $\mathrm{M}^{2}$ \\ ${ }^{1}$ Monash Cardiovascular Research Centre, Monash HEART, Melbourne, Australia \\ ${ }^{2}$ The Prince Charles Hospital, Brisbane, Australia
}

\begin{abstract}
Recent public health reports suggesting a global rise in methamphetamine use among youth. Current data show the most common forms of chronic cardiovascular disease associated with methamphetamine use are cardiomyopathy and coronary artery disease. We report a 39-year-old Caucasian man who was diagnosed with constrictive pericarditis in the setting of modest recreational methamphetamine use by smoking for over ten years. Primarily, his diagnosis was delayed, and the investigations were directed towards a primary liver pathology due to the deceptive history of drug use and clinical symptoms of right heart failure.
\end{abstract}

\section{Introduction}

Emerging evidence reveal methamphetamine (MA) use has substantially grown worldwide and in Australia over the last few years [1]. The association between long term MA abuse and a wide range of cardiovascular disease has been well described [2]. Current data show the most common forms of chronic cardiovascular disease associated with MA use are cardiomyopathy followed by severe coronary artery disease [3]. The pathophysiological mechanism of MA abuse is due to release of endogenous catecholamines (dopamine and noradrenaline), subsequent stimulation and over activation of both alpha and beta-adrenergic receptors. These result in developing hypertension, tachycardia, vasospasm, vasoconstriction and further risk of acute coronary syndrome $[3,4]$. Although several cardiopulmonary complications of MA have been formerly reported [5], the chronic pericardial disease is extremely rare, and it is not well known in this context.

\section{Case report}

We report a 39-year-old Caucasian man who was diagnosed with constrictive pericarditis in the setting of modest recreational methamphetamine use by smoking for over ten years. He did not have a significant medical history or taking prescribed medications. symptomatic with pleuritic chest pain six months prior when he was diagnosed with acute pericarditis, yet transthoracic echocardiogram was unremarkable at the time. Over the next six months, he developed progressive dyspnoea on exertion, abdominal distention and extensive lower limbs pitting oedema. Initially, his diagnosis was delayed due to the deceptive symptoms and history of drug use, and the investigations were directed towards a primary liver pathology.

The diagnosis of constrictive pericarditis was firstly suggested by cardiac imaging studies which revealed a marked pericardial thickening, pericardial tethering, minimum pericardial effusion and a pronounced diastolic interventricular septal bounce (Figure-1). Invasive hemodynamic measurements by the left and right heart catheterisation established the diagnosis of constrictive pericarditis by demonstrating a biventricular elevation and equalisation of diastolic pressure (16 mmHg). Pathology studies include Acid Fast Bacilli (AFB), the QuantiFERON Gold and autoimmune screening tests were negative. Eventually, the patient underwent a successful pericardiectomy and obtained histologic specimen confirmed thickening of the pericardium by dense hyalinized fibrosis consistent with constrictive pericarditis.

\section{Discussion}

To our knowledge, this is the first report of constrictive pericarditis in the setting of recreational MA abuse. Due to the paucity of knowledge in this field, the authors reviewed and collected existing data to identify the possible pathophysiological aetiology of this finding. Several studies demonstrated a compelling association between MA use and increased 5HT (a clinically effective precursor of serotonin) release in the striatum [6]. On the other hand, the role of elevated 5HT in pericardial disease and constrictive pericarditis has been explained in the literature [7]. In this case, we postulated that the long term MA abuse could result in elevated serotonin and further development of chronic pericardial disease and constrictive pericarditis. Quantifying serotonin level was not feasible, due to the absence of a consensus method to measure circulating serotonin and the difficulties in measuring this hormone [8]. The other known causes for constrictive pericarditis have already been excluded in this case.

\section{Conclusion}

This case highlights an extremely rare but debilitating cardiac side effect of recreational MA use. Although pericardial involvement as a side effect of MA use is exremely rare and unsual, awareness of all the potential cardiac complications is warranted to commence an early and appropriate treatment.

Correspondence to: Sam Mirzaee MD, MPH, FRACP, 1Monash Cardiovascular Research Centre, Monash HEART, Melbourne, Australia, E-mail: sammirzaee@ hotmail.com

Key words: methamphetamine use, constrictive pericarditis

Received: December 20, 2017; Accepted: January 11, 2018; Published: January 15,2018 


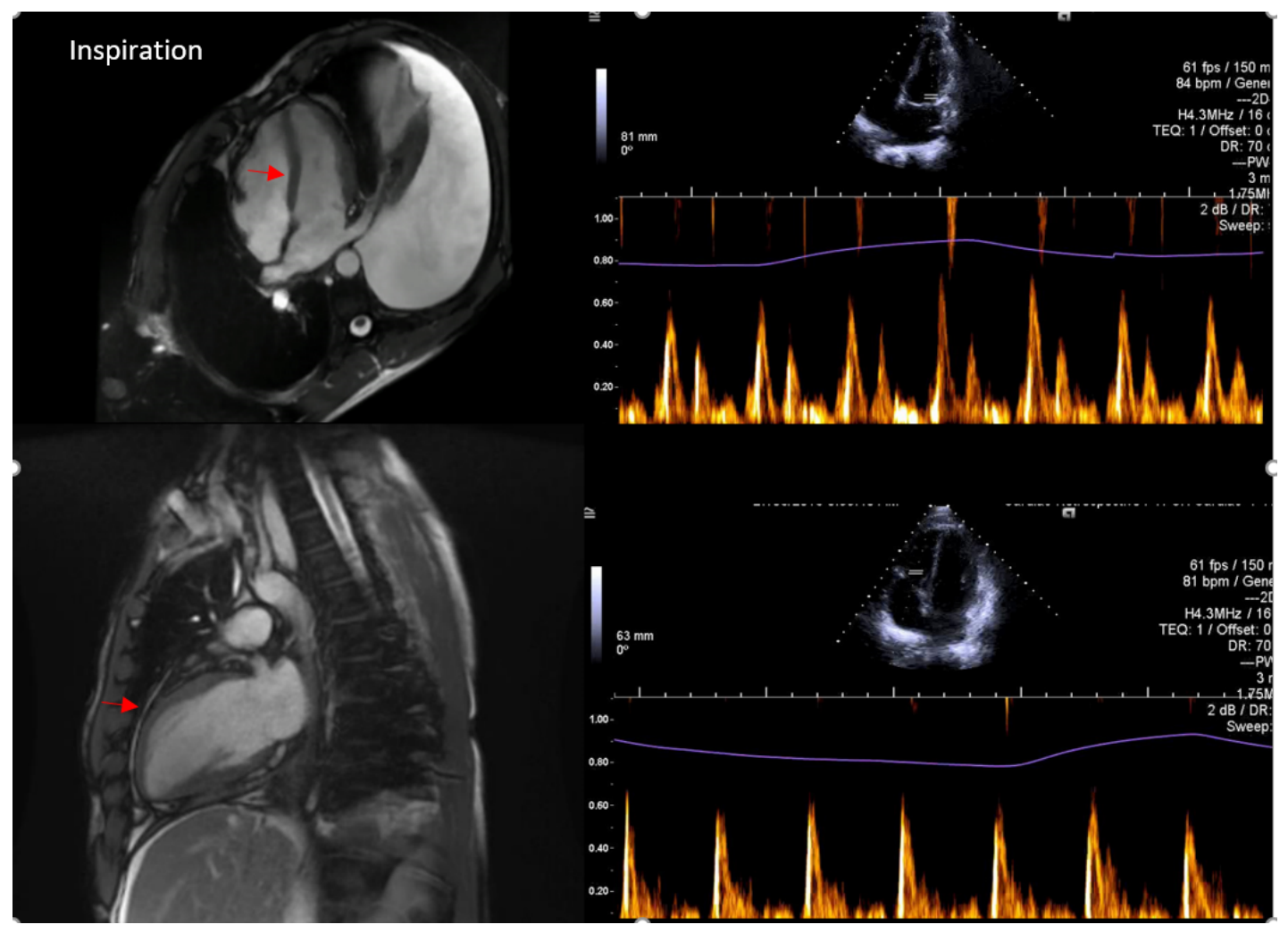

Figure 1. Interventricular septal bounce and pericardial thickening (red arrows)

\section{References}

1. Darke S, Duflou J, Kaye S (2017) Prevalence and nature of cardiovascular disease in methamphetamine-related death: A national study. Drug and Alcohol Dependence 179: 174-179.

2. Huang MC, Yang SY, Lin SK, Chen KY, Chen YY, Kuo CJ, et al (2016) Risk of Cardiovascular Diseases and Stroke Events in Methamphetamine Users: A 10-Year Follow-Up Study. The Journal of Clinical Psychiatry 77: 1396-403.

3. Darke S, Duflou J, Kaye S (2017) Prevalence and nature of cardiovascular disease in methamphetamine-related death: A national study. Drug Alcohol Depend 179:174-179.

4. Kaye S, McKetin R, Duflou J, Darke S (2007) Methamphetamine and cardiovascular pathology: a review of the evidence. Addiction (Abingdon, England) 102:1204-1211.
5. Gotway MB, Marder SR, Hanks DK, Leung JWT, Dawn SK, Gean AD, et al. (2002) Thoracic Complications of Illicit Drug Use: An Organ System Approach. Radio Graphics 22: S119-S35.

6. Thomas DM, Angoa-Pérez M, Francescutti-Verbeem DM, Shah MM, Kuhn DM, et al (2010) The Role of Endogenous Serotonin in Methamphetamine-Induced Neurotoxicity to Dopamine Nerve Endings of the Striatum. Journal of Neurochemistry 115: 595-605.

7. Johnston SD, Johnston PW, O'Rourke D (2014) Carcinoid constrictive pericarditis. Heart 82: 641-643.

8. Lee GS, Simpson C, Sun B-H, Yao C, Foer D, Sullivan B, et al. (2014) Measurement of plasma, serum, and platelet serotonin in individuals with high bone mass and mutations in LRP5. Journal of Bone and Mineral Research: the official journal of the American Society for Bone and Mineral Research 29: 976-98

Copyright: (C2018 Mirzaee S. This is an open-access article distributed under the terms of the Creative Commons Attribution License, which permits unrestricted use, distribution, and reproduction in any medium, provided the original author and source are credited. 